\title{
Correlation between Short-Form 36 Scores and Neck Disability Index in Patients Undergoing Anterior Cervical Discectomy and Fusion
}

\author{
Sangbong Ko, Wonkee Choi, Seungbum Chae, Jaebum Kwon, Youngsik Lee \\ Department of Orthopaedic Surgery, Daegu Catholic University Medical Center, Daegu, Korea
}

\begin{abstract}
Study Design: Case control study.
Purpose: To determine how the Neck Disability Index (NDI), a cervical spine-specific outcome, reflects health-related quality-of-life, and if NDI is correlated to the 36-item Short-Form Health Survey (SF-36) scores.

Overview of Literature: NDI is a useful tool for assessing health-related quality of life in patients with neck pain.

Methods: We used the Pearson product-moment correlation coefficient to assess the validity of all items under NDI and SF-36, and the Pearson's correlation coefficient to assess the correlation between NDI and total SF-36 scores. The primary outcome measures were spine-specific health status- and general health status-measures after spine surgery, and these were evaluated every year for 2 years, using both NDI and SF-36 scores.

Results: NDI had a strong linear correlation with SF-36 and its two scales, the Physical Component Score (PCS) and the Mental Component Score (MCS), attesting to the validity of these two instruments. Among the eight subscales of SF-36, there was a strong linear correlation between NDI and PCS-physical functioning, PCS-bodily pain, and MCS-role emotional. Further, a moderate linear correlation was observed between NDI and subscales of PCS-role physical, PCS-general health, and MCS-social functioning, and between NDI and MCS-vitality and MCS-mental health.

Conclusions: Our findings suggest that the NDI adequately reflects the patient's physical and mental quality of life, implying that the use of NDI to assess functional outcomes can also be ultimately used to evaluate the patient's quality of life.
\end{abstract}

Keywords: Cervical vertebrae; Outcome assessment; Neck Disability Index; Short Form-36

\section{Introduction}

The current trend of increasing number of cervical spine surgeries owing to the aging population, necessitates the identification of clinical outcomes. Specifically, patientreported outcomes and health-related quality of life (HRQOL) measures are frequently used to assess patients health and their response to spine surgery. The primary surgical outcome measures have predominantly been patient-reported outcomes, including condition-specific outcomes [1]. Condition-specific outcomes are important not only for patients but also for clinicians, who regard such clinical information as critical. Assessing a patients' quality of life is also becoming increasingly important,

Received Nov 3, 2017; Revised Nov 29, 2017; Accepted Dec 17, 2017

Corresponding author: Sangbong Ko

Department of Orthopedics, Daegu Catholic University Medical Center, 33 Duryugongwon-ro 17-gil, Nam-gu, Daegu 42472, Korea

Tel: +82-53-650-4283, Fax: +82-53-626-4272, E-mail: bong@cu.ac.kr 
and recently, many studies evaluated the relationship between condition-specific outcomes and HRQOL measures [2]. Chiu et al. [3] have reported a moderate correlation between disability and patient satisfaction and between disability and neck pain, and have also found a weak relationship between pain and patient satisfaction. Therefore, all of these variables should be assessed separately when evaluating the effect of any form of treatment for neck pain $[3,4]$.

The Neck Disability Index (NDI) is one of the widely used tools for evaluating spine-specific outcomes in the neck, and the 36-item Short-Form Health Survey (SF-36) is an effective health status self-assessment tool [3]. NDI is a patient-completed, condition-specific, functional status questionnaire with 10 items that measure disability due to neck pain. Vernon and Mior [5] have reported that the NDI has been used in over 300 publications and translated into 22 languages. They also note that this evaluation tool has been endorsed by a number of clinical practice guideline committees, making it the most widely used and the most strongly validated instrument for assessing disability in patients with neck pain. The questionnaire assesses functional activity (seven items), symptoms (two items), and concentration (one item). A few studies have investigated if the NDI can accurately reflect patient quality of life and overall satisfaction. Disease-specific health measures are used to evaluate patients with disorders at specific sites, and these two instruments, namely NDI and SF-36, are sensitive to specific diseases. To date, however, few studies have examined if disease-specific health information is useful for evaluating overall quality of life. Therefore, the aim of this study was to determine how the NDI, a spine-specific outcome measurement tool, and the SF-36, a general HRQOL questionnaire, correlate with each other.

\section{Materials and Methods}

\section{Patient population}

This study was approved by the institutional review board of Daegu Catholic University Medical Center. (IRB approval no., CR-16-156). We enrolled 133 patients who had undergone one- or two-level anterior cervical discectomy and fusion (ACDF) procedures from January 2013 to December 2014 at at Daegu Catholic University Medical Center, and who were followed up for at least 1

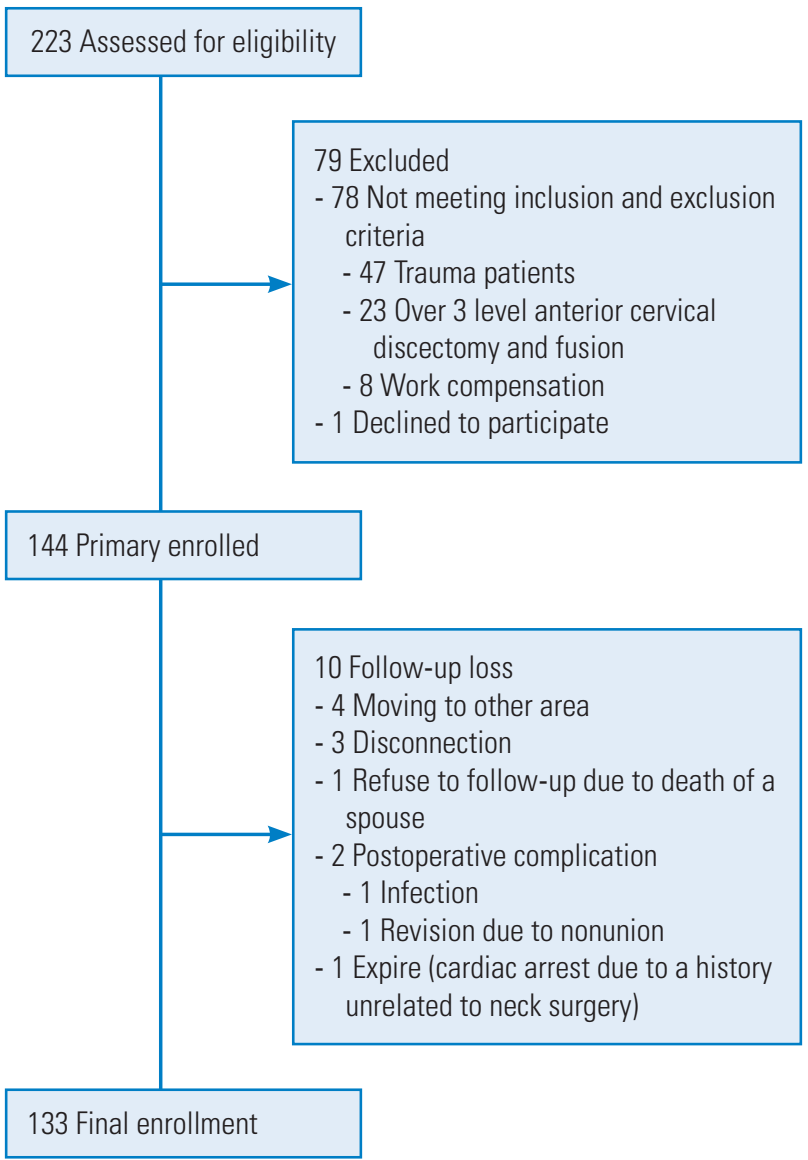

Fig. 1. A flow chart showing patient enrollment.

year after the surgery (Fig. 1). All patients were diagnosed with cervical radiculopathy or cervical spinal myelopathy based on clinical symptoms, magnetic resonance imaging and computed tomography scans, and neurologic tests. Clinical symptoms included neck pain, referred pain, and radiating pain. Radiologic findings included nerve root or spinal cord compression by soft tissue (intervertebral disk or ligamentum flavum) or osteophytes. Following spine surgery by one surgeon (SBK), patients completed the NDI and SF-36 each year to assess their spine-specific and general health status. We excluded patients who obtained secondary gain such as work compensation.

\section{Outcome measurements}

The SF-36 is a patient-reported questionnaire for assessing general HRQOL [6] that has two important summary measures, namely, the Physical Component Score (PCS) and the Mental Component Score (MCS). We derived the raw scores for each of the eight SF-36 dimensions- 

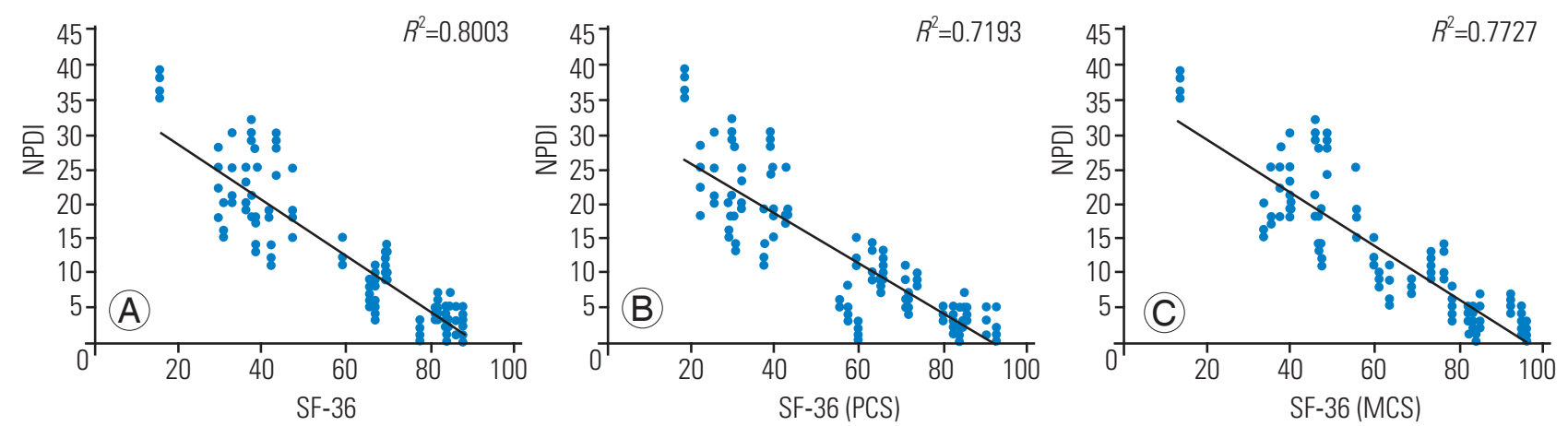

Fig. 2. (A) Correlation between SF-36 and NPDI. (B) Correlations between SF-36 PCS and NPDI. (C) Correlations between SF-36 MCS and NPDI. SF-36, 36-item Short-Form Health Survey; NPDI, Neck Pain Disability Index; PCS, Physical Component Score; MCS, Mental Component Score.

vitality (VT), physical functioning (PF), bodily pain (BP), general health perceptions $(\mathrm{GH})$, role physical (RP), role emotional (RE), social functioning (SF), and mental health $(\mathrm{MH})$-by summing the item scores and converting them to values from 0 to 100 . The NDI consists of 10 items, each with a score up to 5 , for a total score of 100 $(50 \times 2)$; a higher score indicates greater patient-rated neck disability. Unless patients reported that one or two items did not apply to their lives, we scored the instrument on a maximum possible score of 45 , converted the result to $100 \%$, and divided that result by 2 . The minimum clinically important difference was between $5 \%$ and $10 \%$ in each patient [7].

\section{Statistical analyses}

We used the Pearson's product-moment correlation coefficient to assess the validity of each item on both the NDI and SF-36 and applied Pearson's $r$ correlation coefficient to assess the linear correlation between NDI and total SF36 scores. An ' $r$ ' value between 1.0 and 0.7 was defined as a strong correlation, between 0.7 and 0.3 as moderate, and between 0.3 and 0.1 as weak. Coefficient values less than 0.1 were defined as negligible. All statistical analyses were performed using the IBM SPSS software ver. 19.0 (IBM Corp., Armonk, NY, USA), and $p \leq 0.05$ was considered to be statistically significant.

\section{Results}

\section{Correlation between the 36-item Short-Form Health Survey and Neck Disability Index}

SF-36 was significantly correlated with NDI $(r=-0.861$, $p=0.001$ ) (Fig. 2A), and we observed a significant linear correlation between both the PCS and the MCS scores and the NDI (PCS: $r=-0.848, p=0.001$; MCS: $r=-0.879$, $p=0.001$ ) (Fig. 2B, C). Besides the physical and mental status of the patient, the NDI also demonstrated a close correlation with the overall quality of life scores, implying that NDI can also be used to evaluate the patient's quality of life.

\section{Correlations between 36-item Short-Form Health Survey subscales and Neck Disability Index}

We noted strong, statistically significant, linear correlations between NDI and SF-36 PCS subscales of PF and BP ( $r=-0.738, p=0.001$ and $r=-0.726, p=0.001$, respectively) (Fig. 3A, C). In addition, statistically useful, moderate linear correlations were also observed between NDI and VT and GH ( $r=-0.675, p=0.001$ and $r=-0.625, p=0.001$, respectively) (Fig. 3B, D).

Among the physical status measures, NDI showed a strong correlation with physical limitation due to pain, and limitations in the activities of daily living. NDI also showed a moderate correlation with vitality of life and overall health status.

Strong, statistically significant linear correlations were also detected between NDI and the SF-36 MCS subscale of RE ( $r=-0.799, p=0.001)$ (Fig. 3G). Other MCS subscales, namely, SF, VT, and MH were also statistically significant but moderately correlated to NDI $(r=-0.673$, $r=-0.623$, and $r=-0.524$, respectively; all $p=0.001$ ) (Fig. 3E, F, H). Thus, PCS-RP, PCS-BP, and MCS-RE had strong correlations with NDI, and the remaining sub-scales were moderately correlated.

With respect to mental status, NDI scores were strongly 

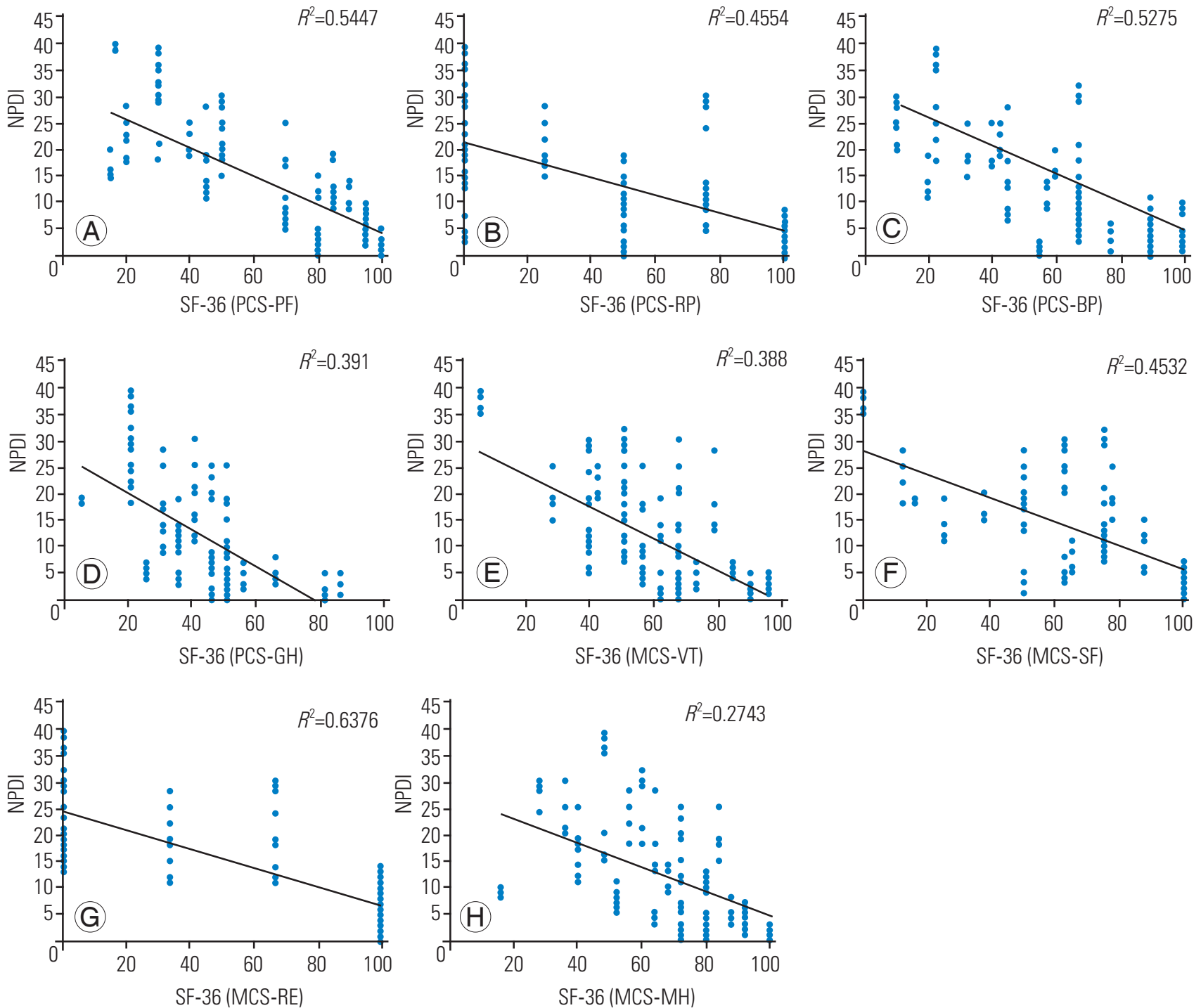

Fig. 3. Correlation between the eight SF-36 subscales and NPDI. (A) Correlation between SF-36 PCS-PF and NPDI. (B) Correlation between SF-36 PCS-RP and NPDI. (C) Correlation between SF-36 PCS-BP and NPDI. (D) Correlation between SF-36 PCS-GH and NPDI. (E) Correlation between SF36 MCS-VT and NPDI. (F) Correlation between SF-36 MCS-SF and NPDI. (G) Correlation between SF-36 MCS-RE and NPDI. (H) Correlation between SF-36 MCS-MH and NPDI. SF-36, 36-item Short-Form Health Survey; NPDI, Neck Pain Disability Index; PCS, Physical Component Score; PF, physical functioning; RP, role physical; BP, bodily pain; GH, general health; MCS, Mental Component Score. VT, vitality; SF, social functioning; RE, role emotional; $\mathrm{MH}$, mental health.

correlated with limitations in tasks or activities of daily living due to emotional distress. The NDI also showed a moderate correlation with impaired social functioning due to the patients' emotional status, or limitations in living due to the patients' vitality or mood.

\section{Discussion}

Self-reported pain and disability are usually the primary focus of neck disorders. NDI is one of the most commonly used self-reportable measures among patients with neck pain $[8,9]$. The author of the scale has also published a summary paper in 2008 summarizing its 17 -year history [10]. MacDermid et al. [11] stated that, among the existing instruments for evaluating neck pain, NDI is the most validated self-report measure, and its reliability and validity have been proven by other studies $[5,12,13]$. NDI was initially applied to evaluate restrictions in the ability to perform daily activities because of severe neck pain, especially following whiplash [5]. Interestingly, NDI was modeled on a similar instrument for assessing self-rated disability in patients with lower back pain, the Oswestry 
Low Back Pain Disability Questionnaire, and the authors of this scale also identified correlations between the Oswestry Disability Index and the SF-36 [2], which is the most valid measure of overall health and quality of life.

Andrade-Ortega et al. [14] have reported that correlations between pain, disability, and HRQOL were uneven, mainly because of the poor correlation between pain changes and changes captured by the HRQOL. The authors also indicated that patient satisfaction with treatment depended clearly on improving pain intensity and associated disability, but that there was a very poor relationship between HRQOL and satisfaction. These results suggest that there is a poor relationship between simple pain and degree of improvement in disability. In contrast, our findings show close correlations between pain, disability, and HRQOL, given that SF-36 PCS scores and quality of life scores had a strong linear correlation with the NDI ( $r=0.747)$. Interestingly, these results are consistent with those of Song et al. [15], who demonstrated a stronger correlation between NDI and both the PCS-PF and the PCS-BP than with either the PCS-GH or the PCS$\mathrm{RP}$, even though adding the PCS-GH and PCS-RP subscales is thought to aid in evaluating quality of life.

Although MacDermid et al. [11] indicated that the NDI lacks psychometric evidence, our findings reveal a strong linear correlation with the SF-36 MCS, contrary to the assertions of MacDermid et al. [11]. In particular, MCS-RE had a stronger correlation with the NDI than did the SF, the VT, or the MH sub-scales.

Cook et al. [16] have reported strong correlations between NDI and SF-36 PCS-PF and MCS-SF sub-scales, whereas Song et al. [15] found that NDI correlated with SF-36 sub-scales of PCS-BP and PCS-GH. The study by Cook et al. [16] used data from 203 patients with neck bruising, cervical spine fracture, and degenerative arthritis, whereas Song et al. [15] carried out their study in 60 patients with degenerative cervical spine disease, cervical herniated disk with neuropathy, and cervical myelopathy.

In our study, we enrolled 133 patients who underwent ACDF for cervical radiculopathy or myelopathy, and thus, there are differences in patient composition between our study and the two studies discussed above. Furthermore, whereas Cook et al. [16] focused mainly on assessing spine-specific outcome measures in patients with trauma, we did not assess either the effects of secondary gains such as work compensation in these patients or other general aspects of patients with neck disorders.
MacDermid et al. [11] have stated that because the NDI was not developed using a clinimetric process and because the original pilot testing was conducted on a very small sample size, it is not clear whether NDI adequately captures all the important factors affecting patients with neck pain. Additionally it is also not known if it weighs pain and disability based on their relative priority, which leaves open the possibility that adding items to the scale can enhance its performance. Contrarily, Van der Velde et al. [17] have suggested that removing items might improve performance. Together, these reports imply that there are gaps in defining clinically useful comparative data and patients' evaluation.

NDI and SF-36 are instruments designed to assess disease-specific outcomes and the overall quality of life, and they each have both strengths and limitations. Nonetheless, till date, there are growing doubts on the clinical significance of NDI and whether it can assess patient quality of life. Encouragingly, we found strong linear correlations between the NDI and the SF-36 in terms of consistent clinical outcomes, implying that it is an effective instrument for assessing patients' quality of life. These results notwithstanding, our study has some limitations. Although both the questionnaires have been fully validated $[18,19]$, there may have been differences in translating them into a culturally-appropriate Korean version. Separately, in the context of this study, the inclusion of only one-level and two-level ACDF patients may not accurately reflect the validity of the NDI as a general health measure.

\section{Conclusions}

Based on the results of our study, we propose that the NDI suitably reflects SF-36 PCS-BP, PCS-PF, and MCS-RE scores, and that it also fully reflects the physical and mental aspects of patients' quality of life after neck surgery. Furthermore, using the NDI to assess functional status may ultimately lead to its use in the clinical evaluation of HRQOL in patients with neck pain.

\section{Conflict of Interest}

No potential conflict of interest relevant to this article was reported. 


\section{ORCID}

Sangbong Ko: https://orcid.org/0000-0003-3527-9251

Wonkee Choi: https://orcid.org/0000-0002-4671-5656

Seungbum Chae: https://orcid.org/0000-0003-3469-9289

Youngsik Lee: https://orcid.org/0000-0002-4663-1495

\section{References}

1. Bombardier C. Outcome assessments in the evaluation of treatment of spinal disorders: summary and general recommendations. Spine (Phila Pa 1976) 2000;25:3100-3.

2. Ko S, Chae S. Correlations between the SF-36, the Oswestry-Disability Index and Rolland-Morris Disability Questionnaire in patients undergoing lumbar decompression according to types of spine origin pain. Clin Spine Surg 2017;30:E804-8.

3. Chiu TT, Lam TH, Hedley AJ. Correlation among physical impairments, pain, disability, and patient satisfaction in patients with chronic neck pain. Arch Phys Med Rehabil 2005;86:534-40.

4. DeVine J, Norvell DC, Ecker E, et al. Evaluating the correlation and responsiveness of patient-reported pain with function and quality-of-life outcomes after spine surgery. Spine (Phila Pa 1976) 2011;36(21 Suppl):S69-74.

5. Vernon H, Mior S. The Neck Disability Index: a study of reliability and validity. J Manipulative Physiol Ther 1991;14:409-15.

6. Ware JE, Kosinski M, Keller SD. SF-36 physical and mental health summary scales: a user's manual. Boston (MA): Health Assessment Lab; 1994.

7. Howell ER. The association between neck pain, the Neck Disability Index and cervical ranges of motion: a narrative review. J Can Chiropr Assoc 2011;55:21121.

8. Pietrobon R, Coeytaux RR, Carey TS, Richardson WJ, DeVellis RF. Standard scales for measurement of functional outcome for cervical pain or dysfunction: a systematic review. Spine (Phila Pa 1976) 2002;27:515-22.
9. Resnick DN. Subjective outcome assessments for cervical spine pathology: a narrative review. J Chiropr Med 2005;4:113-34.

10. Vernon H. The Neck Disability Index: state-ofthe-art, 1991-2008. J Manipulative Physiol Ther 2008;31:491-502.

11. MacDermid JC, Walton DM, Avery S, et al. Measurement properties of the neck disability index: a systematic review. J Orthop Sports Phys Ther 2009;39:400-17.

12. Hains F, Waalen J, Mior S. Psychometric properties of the neck disability index. J Manipulative Physiol Ther 1998;21:75-80.

13. Miettinen T, Leino E, Airaksinen O, Lindgren KA. The possibility to use simple validated questionnaires to predict long-term health problems after whiplash injury. Spine (Phila Pa 1976) 2004;29:E47-51.

14. Andrade-Ortega JA, Ceron-Fernandez E, RibeiroGonzalez M, Garcia-Llorent R, Almecija-Ruiz R, Delgado-Martinez AD. Pain, disability and quality of life in chronic nonspecific neck pain. Phys Med Rehabil Int 2016;3:1094.

15. Song KJ, Choi BW, Choi BR, Seo GB. Cross-cultural adaptation and validation of the Korean version of the neck disability index. Spine (Phila Pa 1976) 2010;35:E1045-9.

16. Cook C, Richardson JK, Braga L, et al. Cross-cultural adaptation and validation of the Brazilian Portuguese version of the Neck Disability Index and Neck Pain and Disability Scale. Spine (Phila Pa 1976) 2006;31:1621-7.

17. Van der Velde G, Beaton D, Hogg-Johnston S, Hurwitz E, Tennant A. Rasch analysis provides new insights into the measurement properties of the neck disability index. Arthritis Rheum 2009;61:544-51.

18. Lee H, Nicholson LL, Adams RD, Maher CG, Halaki M, Bae SS. Development and psychometric testing of Korean language versions of 4 neck pain and disability questionnaires. Spine (Phila Pa 1976) 2006;31:1841-5.

19. Lee JS, Suh KT, Kim JI, Lee HS, Goh TS. Validation of the Korean version of the neck pain and disability scale. Asian Spine J 2013;7:178-83. 